\title{
NUMERICAL SIMULATIONS OF WAVE BREAKING
}

\author{
Philippe Helluy ${ }^{1}$, Frédéric Golay ${ }^{1}$, Jean-Paul Caltagirone ${ }^{2}$, Pierre Lubin ${ }^{2}$, \\ Stéphane Vincent ${ }^{2}$, Deborah Drevard ${ }^{3}$, Richard Marcer ${ }^{4}$, Philippe Fraunié ${ }^{3}$, \\ Nicolas Seguin $^{5}$, Stephan Grilli ${ }^{6}$, Anne-Cécile Lesage ${ }^{7}$, Alain Dervieux ${ }^{7}$ \\ AND Olivier Allain ${ }^{8}$
}

\begin{abstract}
This paper is devoted to the numerical simulation of wave breaking. It presents the results of a numerical workshop that was held during the conference LOMA04. The objective is to compare several mathematical models (compressible or incompressible) and associated numerical methods to compute the flow field during a wave breaking over a reef. The methods will also be compared with experiments.
\end{abstract}

Mathematics Subject Classification. 65M12, 74S10.

Numerical workshop, Low Mach Number Flows Conference, June 21-25, 2004, Porquerolles, France.

\section{INTRODUCTION}

In this paper, we present and compare several numerical methods to compute the breaking of a stable solitary wave over a submerged rectangular reef. This test case has been first proposed by Yasuda, Mutsuda and Mizutani in [39], together with physical experiments.

Generally, air and water are supposed incompressible in this kind of flows. However, for several reasons, it is interesting to envisage the more general compressible model.

- For physical reasons: in some parts of the flow, compressible effects may be not negligible. For example, at the reconnection point of the water, or in the trapped air bubble.

- For numerical reasons: incompressible solvers require an implicit resolution of the pressure equation. Compressible solvers can be completely explicit. Even if the starting model is incompressible it can thus be useful to add an artificial numerical compressibility.

\footnotetext{
Keywords and phrases. Wave breaking, finite volumes, low Mach compressible flows, multiphase flow.

1 ISITV/MNC, BP 56, 83162 La Valette cedex, France.

2 TREFLE - ENSCPB - UMR CNRS 8508, Bordeaux, France.

3 LSEET, Université de Toulon, BP 132F, La Garde Cedex, France.

4 Principia Z.I. Athélia 13705 La Ciotat Cedex, France.

5 Laboratoire J.-L. Lions, Université Paris VI, France.

6 Department of Ocean Engineering, University of Rhode Island, Narragansett, RI 02882, USA.

7 INRIA, Sophia Antipolis, France.

8 Société Lemma, La Roquette-Sur-Siagne, France.
} 


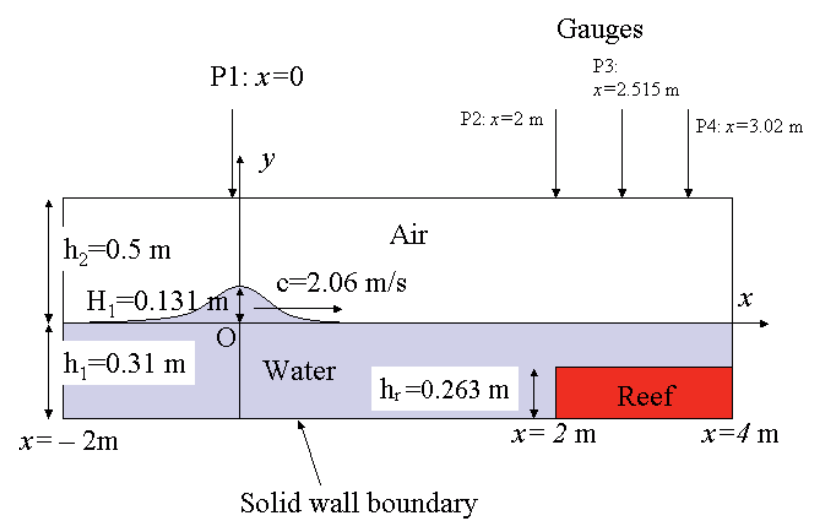

Figure 1. Boundary and initial conditions.

- For practical reasons: the all-purpose-solver is an utopia. But it is interesting to have one solver for all flow regime. There is less programmation effort. Of course, it is probable that a compressible solver will be more expensive than specialized solvers in many situations. There is also a very important practical reason: a comparison between different solvers is crucial for the validation of the physical and numerical models.

Of course, a compressible solver has to face the well known loss of precision due to the low Mach number of the flow. About this subject we refer for example to [20,31,32] and included references. This loss of precision is mainly due to the upwinding of compressible solvers. The upwinding introduces an artificial viscosity proportional to the sound speed of the flow. If the Mach number is small, the low speed waves, moving with the material velocity, are too much damped and the precision becomes insufficient. The technique of preconditioning aims at diminishing the numerical viscosity, keeping the stability of the scheme.

This paper has been written in the context of a conference about low Mach number flows. But the computation of wave breaking has a long history. This history and the more general context are briefly recalled in section 5 .

The objective here is to compare several models of wave breaking and their associated numerical methods, based on incompressible or compressible solvers. We present the results of the numerical workshop "Free Surface Flow" of the conference "Mathematical and Numerical aspects of Low Mach Number Flows" that was held in Porquerolles (France) in June 2004. The web site of the conference is at

http://www-sop.inria.fr/smash/LOMA/

The web site of the numerical workshop is at

http://helluy.univ-tln.fr/soliton.htm .

The boundary and initial conditions of the proposed test case are sketched on Figure 1. The initial condition is a stable incompressible solitary wave computed thanks to the method of Tanaka [30]. The still water level is at $h_{1}=0.31 \mathrm{~m}$ over the bottom. The crest of the solitary wave is at $H_{1}=0.131 \mathrm{~m}$ over the still water level. It propagates at a phase velocity $c=2.06 \mathrm{~m} / \mathrm{s}$, see Figure 1 . The horizontal and vertical components of the velocity at time $t=0$ are plotted on Figures 2 and 3 .

The programs that compute the free surface profile and the initial velocity field can be downloaded at

http://helluy.univ-tln.fr/soliton.htm .

More precise indications on the test case are given on the above mentioned web page. 


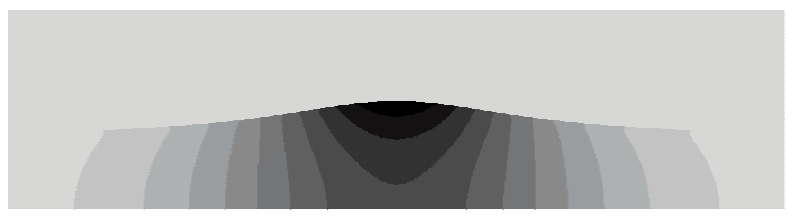

Figure 2. Component $u$ of the velocity at time $t=0$.

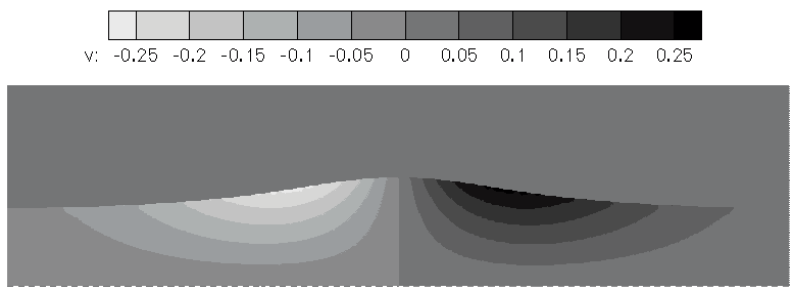

Figure 3. Component $v$ of the velocity at time $t=0$.

The organization of the paper is then as follows.

First, the participants to the workshop propose a short overview of the model and the numerical method they used (Sects. 1 to 6$)$. The different contributors are:

- P. Helluy and F. Golay: the model is a compressible multi-fluid model. The method is the finite volume method with an exact Riemann solver. The preconditioning is obtained thanks to a modification of the physical sound speed.

- P. Lubin, S. Vincent and J.-P. Caltagirone: the model is made of the incompressible Navier-Stokes equations coupled with the advection of an indicator function in order to distinguish the two phases. The Navier-Stokes solver is based on the finite volume method on a staggered grid and an augmented Lagrangian approach. It is coupled with a TVD scheme for the advection of the indicator function.

- D. Drevard, R. Marcer and P. Fraunié: the model is made of the incompressible Navier-Stokes equations in the liquid phase coupled with a tracking of the free surface. The numerical method is based on a pseudo-compressibility formulation [37]. The interface is located with a second order version of the VOF method of Hirt and Nichols [21].

- N. Seguin: the model is made of the one-dimensional shallow water equations with topography. The numerical method uses a well-balanced finite volume scheme and can handle non-smooth topography.

- S. Grilli: the model is based on a non-stationary, non-linear boundary integral equation. It is discretized by the Boundary Integral Element Method. The breaking can be computed until the reconnection.

- A.-C. Lesage, A. Dervieux and O. Allain: the model is the incompressible Navier-Stokes equation coupled with the evolution of a level-set function. They are solved by a high order projection method and a high order convection scheme respectively. The mesh is 3D (with 2 mesh points in the third direction), with possibility of mesh refinement.

Obviously, the place was not sufficient to describe in detail all the methods. For more informations, we refer to the bibliographic references. 
In Section 7, the different numerical methods will then be assessed through comparisons, including comparisons with experiments.

\section{Over-compressible multiphase model (Golay, Helluy)}

\subsection{Mathematical model}

We consider a 2D, compressible, two-fluids flow model. The unknowns depend on the spatial position $(x, y)$, the time $t$ and are the density $\rho(x, y, t)$, the two components of the velocity $u(x, y, t), v(x, y, t)$, the internal energy $\varepsilon(x, y, t)$, the pressure $p(x, y, t)$ and the fraction of water $\varphi(x, y, t)$. The fraction satisfies $0 \leq \varphi \leq 1$, $\varphi=1$ in the water and $\varphi=0$ in the air. Considering the gravity $g$, neglecting viscous effects and superficial tension, but keeping compressible effects, we propose to consider the following Euler equations:

$$
\begin{aligned}
& \rho_{t}+(\rho u)_{x}+(\rho v)_{y}=0 \\
& (\rho u)_{t}+\left(\rho u^{2}+p\right)_{x}+(\rho u v)_{y}=0 \\
& (\rho v)_{t}+(\rho u v)_{x}+\left(\rho v^{2}+p\right)_{y}=-\rho g \\
& (\rho E)_{t}+((\rho E+p) u)_{x}+((\rho E+p) v)_{y}=-\rho g v \\
& E=\varepsilon+\frac{1}{2}\left(u^{2}+v^{2}\right) \\
& \varphi_{t}+u \varphi_{x}+v \varphi_{y}=0 \\
& p=p(\rho, \varepsilon, \varphi)
\end{aligned}
$$

In this model, the pressure depends on the fraction. In this way, it is possible to distinguish between the liquid and the gas. We have to provide an adequate Equation Of State (EOS). Let $c(x, y, t)$ denotes the sound speed. It is usually admitted in physics that a flow is incompressible if the Mach number $M=\frac{\sqrt{u^{2}+v^{2}}}{c}$ is lower than 0.1. Here, the real (physical) Mach number is much smaller, of the order of $1 / 400 \sim 1 / 1600$. ${ }^{c}$ This is very constraining if an explicit finite volume solver is used because the time step $\Delta t$ has to satisfy a CFL condition of the form

$$
\Delta t \leq \alpha \frac{\Delta x}{\sqrt{u^{2}+v^{2}}+c}
$$

where $\alpha$ is the CFL number, $\Delta x$ the space step and $c$ the sound speed. furthermore, it is known that numerical imprecision also arise due to the low Mach number of the flow. For those two reasons we have been led to chose an artificial pressure law where the sound speed is approximately fixed to $20 \mathrm{~m} \cdot \mathrm{s}^{-1}$. A very simple choice of EOS is the so called stiffened gas EOS that reads

$$
\begin{aligned}
& p=(\gamma(\varphi)-1) \rho \varepsilon-\gamma(\varphi) \pi(\varphi) \\
& \frac{1}{\gamma(\varphi)-1}=\varphi \frac{1}{\gamma_{w}-1}+(\varphi-1) \frac{1}{\gamma_{a}-1} \\
& \frac{\gamma(\varphi) \pi(\varphi)}{\gamma(\varphi)-1}=\varphi \frac{\gamma_{w} \pi_{w}}{\gamma_{w}-1}+(\varphi-1) \frac{\gamma_{a} \pi_{a}}{\gamma_{a}-1}
\end{aligned}
$$

This EOS is very similar to the perfect gas EOS. It has been used by several authors for multi-fluid flows computations $[1,28]$, etc. The pressure law coefficients, $\gamma$ and $\pi$ depend on the fraction $\varphi$. The dependence in (3) will be justified below. The sound speed for this EOS is given by

$$
c=\sqrt{\frac{\gamma(p+\pi)}{\rho}} .
$$


We arbitrarily choose $\gamma_{w}=\gamma_{a}=1.1$ for the air (a) and water (w). On the other hand, if the sound speed is fixed to $20 \mathrm{~m} \cdot \mathrm{s}^{-1}$ for a pressure of $p=10^{5} \mathrm{~Pa}$, we get

$$
\begin{aligned}
& \pi_{a}=-0.99636 \times 10^{5} \mathrm{~Pa}, \\
& \pi_{w}=2.63636 \times 10^{5} \mathrm{~Pa} .
\end{aligned}
$$

\subsection{Numerical method}

The numerical method is a simple second order explicit MUSCL finite volumes method applied to the system (1) and (3). We used an exact Riemann solver because we experimented instabilities with several approximate solvers. It is known that the transport equation on $\varphi$ in (1) has to be approximated with care in order to avoid pressure oscillations. Indeed, the transport equation in (1) and the EOS (3) can be written under many different forms on the continuous side. On the discrete side, these forms are not equivalent, and the one that is presented in (1), (3) plays a special role. We used the trick of Abgrall and Saurel described in $[1,28]$ in order to get a numerical scheme that preserves the constant velocity and pressure states.

\section{Incompressible solver (Lubin, Vincent, Caltagirone)}

\subsection{Single fluid formulation of the Navier-Stokes equations}

Let us consider the wave propagation problem as a two-phase flow involving a liquid phase (water) and a gaseous phase (air).

A general Navier-Stokes model for all fluids is designed by convolving the incompressible Navier-Stokes equations in each phase and the jump conditions across the interface by an indicator function $C$ and by filtering the set of equations thanks to a volume integral operator. This function characterizes one of the fluids, water for example, assuming the value 1 in the water phase and 0 in the others. The air phase is directly obtained as the complementary $1-C$ of the water phase. Assuming the interface between the fluids as the discontinuity of the indicator function, it is practically located by the $C=0.5$ isoline. Several correlation terms are discarded in the single fluid model by making the assumptions that the sliding between phases is negligible and that no phase change occurs. The corresponding free surface flow admits a continuous velocity field through the free surface and is locally isovolume even though $\rho$ may be discontinuous.

Let $\mathbf{u}$ be the velocity field, $\mathbf{g}$ the gravity vector, $\mathrm{p}$ the pressure, $\sigma$ the surface tension, $\kappa$ the curvature, $\mu$ the viscosity and $\rho$ the density. The governing equations for the turbulence model in large eddy simulation of an incompressible fluid flow are classically derived by applying a convolution filter to the unsteady Navier-Stokes equations. In a uniform Cartesian coordinate system $(x, z)$, associated with a bounded domain $\Omega$, the one-fluid model can be expressed as follows:

$$
\begin{gathered}
\mu=\mu_{1}, \rho=\rho_{1} \text { if } C>0.5, \\
\mu=\mu_{0}, \rho=\rho_{0} \text { if } C<0.5, \\
\nabla \cdot \mathbf{u}=0, \\
\rho\left(\frac{\partial \mathbf{u}}{\partial t}+(\mathbf{u} \cdot \nabla) \mathbf{u}\right)=\rho \mathbf{g}-\nabla p+\nabla \cdot\left[\left(\mu+\mu_{t}\right)\left(\nabla \mathbf{u}+\nabla^{T} \mathbf{u}\right)\right]+\sigma \kappa \delta_{i} \mathbf{n}_{i}, \\
\frac{\partial C}{\partial t}+\mathbf{u} \cdot \nabla C=0,
\end{gathered}
$$

where $\delta_{i}$ is a Dirac function indicating the interface, $\mathbf{n}_{i}$ is the unit normal to the interface and $\rho_{0}, \rho_{1}, \mu_{0}$ and $\mu_{1}$ are the respective densities and viscosities in each phase. Equations (6) and (7) correspond to a discontinuous estimate of the physical characteristics. We proved this method to be less diffusive than the usual linear formula used in the literature [33].

The turbulent viscosity $\mu_{t}$ is calculated with the Mixed Scale model, assuming the following relation (11):

$$
\mu_{t}=\rho C_{m} \bar{\Delta}^{\frac{3}{2}} \sqrt{2(\nabla \mathbf{u} \otimes \nabla \mathbf{u})}\left(q_{c}^{2}\right)^{\frac{1}{2}} .
$$


with $C_{m}$ being a constant of the model and $\bar{\Delta}=\left(\Delta_{x} \Delta_{z}\right)^{\frac{1}{2}}$ being the cut-off length scale of the filter, with $(x, z)$ defined as, respectively, the longitudinal and vertical coordinates. The quantity $q_{c}$ represents the kinetic energy of the test field extracted from the resolved velocity field through the application of a test filter associated to the cut-off length scale. All the details about large eddy simulation can be found in [27].

The evolution of the free surface and the physical characteristics of the fluids are simultaneously represented by means of equations (6)-(10). The free surface flow is analyzed in terms of an equivalent single fluid whose variable properties $\rho$ and $\mu$ are related to $\rho_{0}, \rho_{1}, \mu_{0}$ and $\mu_{1}$ of the two real phases by the color function C.

\subsection{Numerical methods}

\subsubsection{Interface capturing method and surface tension discretization}

All interface capturing methods are dedicated to solving the advection equation of a discontinuous indicator function $\mathrm{C}$ through the reformulation of the transport equation with a smooth function. We choose to implement the explicit Lax-Wendroff TVD (LWT) time-stepping scheme dedicated to volume fraction advection, thoroughly explained in Vincent and Caltagirone [34,35]. This approach allows the accurate solution of free-surface flows with strong tearing and stretching of the interface which would occur in breaking water waves.

Due to the volumetric representation of the interface, the geometric interface properties $\kappa, \delta_{i}$ and $\mathbf{n}_{i}$ are not directly accessible. To avoid explicitly calculating these free surface properties, they are modeled as a function of the volume fraction. The Continuum Surface Force (CSF) method of Brackbill et al. [4] is used to model the surface tension acting in the Navier-Stokes equations (9).

\subsubsection{Navier-Stokes solver}

A Finite-Volume method on a staggered mesh is carried out to discretize the Navier-Stokes equations and an augmented Lagrangian technique is employed to solve the coupling between pressure and velocity in the equations of motion (8)-(9).

The discretization of these equations is achieved through a second-order Euler scheme, or GEAR scheme, for the time derivatives while a second order Hybrid Centered-Upwind scheme is applied to the non-linear convective terms and a second order centered scheme is chosen for the approximation of the viscous and the augmented Lagrangian terms. The linear system resulting from the previous implicit discretization is solved with an iterative BiCGSTAB (Bi-Conjugate Gradient Stabilized) algorithm, preconditioned by a Modified and Incomplete LU (MILU) algorithm. References concerning these numerical techniques can be found in [34,35].

\section{Pseudo-compressibility (Drevard, Marcer, Fraunié)}

\subsection{Mathematical formulation}

We consider a moving interface defined as the limit between two incompressible viscous fluids of different densities. The problem is to compute the pressure and velocity fields in the denser fluid and to track the interface. Then, the unsteady 3D Navier-Stokes equations are solved in the denser fluid and are written in the following semi-conservative form, in curvilinear formulation,

$$
\frac{1}{J} \frac{\partial W}{\partial t}+\frac{\partial F}{\partial \xi}+\frac{\partial G}{\partial \eta}+\frac{\partial H}{\partial \chi}=\frac{R}{J}+\frac{T}{J}
$$


where $\mathrm{F}, \mathrm{G}$ and $\mathrm{H}$ are respectively the convective, diffusive and pressure flux terms, $\mathrm{R}$ is the volumic force source term, T the surface tension source term and $J$ the Jacobian of the co-ordinates transformation.

\subsection{Numerical method}

\subsubsection{Pseudo-compressibility method}

Time discretization in the Navier-Stokes model is based on a fully implicit second-order finite difference scheme. The solution of the nonlinear system at time step $n+1$ is based on the "pseudo-compressibility method" (Viviand 1980 [37], De Jouëtte et al. 1991 [5]). In this method, a time-like variable $\tau$, called pseudotime, is introduced in equation (12). This adds pseudo-unsteady terms, which are derivatives of the unknowns at time level $n+1$, with respect to $\tau$. The pseudo-unsteady terms involve a new unknown $\tilde{\rho}$, called pseudo-density, which is constrained to remain positive. The pressure is calculated as a function of $\tilde{\rho}$ through an additional pseudo-state equation,

$$
p^{n+1}=f\left(\tilde{\rho}^{n+1}\right) .
$$

The choice of an optimal pseudo-state equation is discussed in Viviand (1995) [38]. The new system of equations is integrated step-by-step in pseudo-time variable, with an explicit five step Runge-Kunta scheme, associated with an implicit residual smoothing technique.

\subsubsection{Interface tracking method}

For each time step the interface and its evolution are obtained by an original method, called SL-VOF (Guignard et al. 2001 [19] for 2D-flows, Biausser et al. 2004 [3] for 3D-flows), based on both VOF (Hirt and Nichols 1981 [21]) and PLIC (Li 1995 [23]) concepts.

The general algorithm contains three parts. (i) The interface tracking: a color function is defined within each cell of the VOF grid, as the volumic fraction (0 to 1) of the cell area filled with the denser fluid (VOF concept). Then, a multi-segmental representation of the interfaces is defined based on the values of the color function, according to PLIC concept. (ii) The interface advection: interface segments are advected, as a function of time, based on Lagrangian markers, following the velocity field obtained from the solution of NS equations. (iii) The reconstruction of the new VOF field: after the advection, new values of the color function are computed, which take into account the new position of the interface segments.

\section{Shallow Water model (Seguin)}

\subsection{Mathematical model}

In this section, we consider the shallow-water model with topography. This 1D model writes

$$
\left\{\begin{array}{l}
h_{t}+(h u)_{x}=0, \\
(h u)_{t}+\left(h u^{2}+g h^{2} / 2\right)_{x}=-g h a^{\prime}(x),
\end{array}\right.
$$

where $h$ is the height of water, $u$ is the horizontal velocity of water and $a^{\prime}(x)$ is the slope of the bottom, see Figure 4 .

This model is a simplification of the 2D Navier-Stokes equations. The water is considered as an incompressible fluid, the pressure is assumed to be hydrostatic and we suppose that the flow is stratified, which enables to average the $2 \mathrm{D}$ model with respect to $y$, leading to (14). It is clear that for the current test case, the latter assumption is only fulfilled until the wave breaks over the reef. Moreover, this model being fully nonlinear, the initial shape of the solitary wave cannot be preserved. In fact, the left part becomes a rarefaction wave and the right part turns to be a shock wave. Nonetheless, such a model can be very interesting if it is understood as a first approximation of the solution and for simple and very fast numerical simulations. As mentioned above, this model is one-dimensional and thus, the initial data must be adapted. Actually, the initial height of water $h$ 


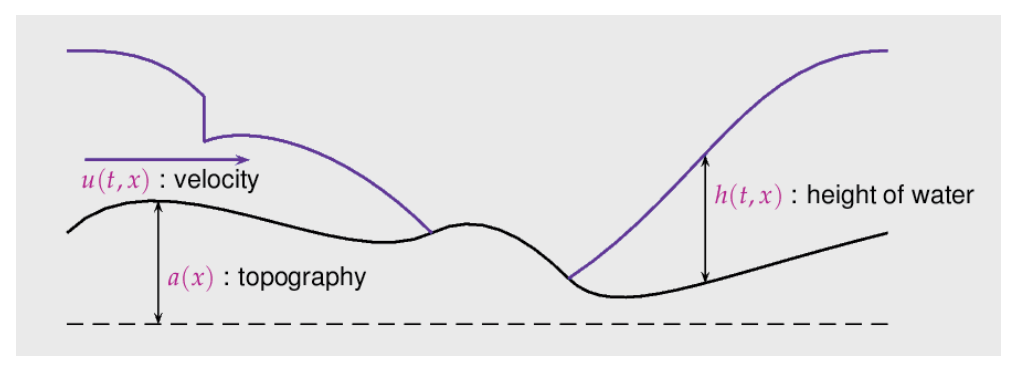

Figure 4. Description of the shallow-water model.

is given by the locus of the interface between water and air and the velocity $u$ is null far from the initial wave and, inside the wave, we computed

$$
u_{0}(x)=-2\left(\sqrt{g h_{1}}-\sqrt{g h_{0}(x)}\right)
$$

At the limit of the domain, the classical mirror state method has been implemented to model solid wall boundaries. The results which are presented have been computed using a mesh with 500 cells.

\subsection{Numerical method}

The numerical method used here has been presented in [7]. It is based on an approximate Riemann solver which includes the source term. Indeed, the numerical fluxes are computed using an approximate solution of each local Riemann problem

$$
\begin{cases}a_{t}=0, & t>t^{n}, x \in \mathbb{R}, \\ h_{t}+(h u)_{x}=0, & t>t^{n}, x \in \mathbb{R}, \\ (h u)_{t}+\left(h u^{2}+g h^{2} / 2\right)_{x}+g h a_{x}=0, & t>t^{n}, x \in \mathbb{R}, \\ (a, h, h u)\left(t^{n}, x\right)= \begin{cases}\left(a_{i}, h_{i}^{n}, h_{i}^{n} u_{i}^{n}\right) & \text { if } x<x_{i+1 / 2}, \\ \left(a_{i+1}, h_{i+1}^{n}, h_{i+1}^{n} u_{i+1}^{n}\right) & \text { if } x>x_{i+1 / 2} .\end{cases} \end{cases}
$$

Let us note that this method is well adapted to simulate steady states, as the flow is far from the solitary wave. The method is explicit and the time step is defined by

$$
\Delta t^{n}=0.4 \frac{\Delta x}{\max _{i}\left(\left|u_{i+1 / 2}^{n}\right|+\sqrt{g h_{i+1 / 2}^{n}}\right)},
$$

where the values ()$_{i+1 / 2}^{n}$ correspond to the approximate solution of each local Riemann problems (15). It is worth noting that the time step does not tends to zero when the source term becomes stiff.

\section{INCOMPRESSIBLE INVISCID IRROTATIONAL FLOW SOLVER (GRILli)}

\subsection{Fully nonlinear potential flow in a BEM formulation}

Numerical models based on the Boundary Element Method (BEM), combined to an explicit higher-order Lagrangian time stepping, have proved very efficient and accurate for solving fully nonlinear potential flow (FNPF) equations with a free surface, in two- $(2 \mathrm{D})$ and three-dimensions $(3 \mathrm{D})([8-11,14])$. When applied to the modeling of surface wave generation and propagation over varying topography, such models have recently been referred to as Numerical Wave Tanks (NWT). Due to their simplicity as compared to periodic waves, 
solitary waves have often been used for both model development and experimental validation. Grilli et al. $[12,13,15-17]$, for instance, showed that the shape of shoaling and breaking solitary waves propagating over a step, a submerged trapezoidal obstacle, or a slope, could be simulated within a few percent of experimental measurements in a 2D-BEM-FNPF-NWT. Similar validations were repeated in 3D, e.g., by Grilli et al. [11]. Both potential flow equations and BEM models, however, break down after impact of the breaker jet on the free surface.

Recently, Volume of Fluid (VOF) models solving Navier-Stokes (NS) equations with a free surface have been used to model breaking waves (e.g., $[19,24,34])$. Such models, however, are much more computationally intensive than BEM models and suffer from numerical diffusion over long distances of propagation. Hence, a coupled approach was recently proposed, for both $2 \mathrm{D}$ and $3 \mathrm{D}$ problems, in which wave generation and shoaling is simulated in a BEM-NWT up to a point close to breaking, and a VOF model is initialized using results of the BEM model, to pursue computations beyond breaking over a finely discretized grid. Wave breaking and post-breaking were thus computed in the VOF model $[2,3,18,22]$.

The 2D-BEM-FNPF model developed by Grilli et al. $[9,10,14]$ is used in these simulations. The velocity potential $\phi(\boldsymbol{x}, t)$ is used to describe inviscid irrotational flows in the vertical plane $(x, z)$ and the velocity is defined by, $\boldsymbol{u}=\boldsymbol{\nabla} \phi=(u, w)$. Continuity equation in the fluid domain $\Omega(t)$ with boundary $\Gamma(t)$ is a Laplace's equation for the potential,

$$
\nabla^{2} \phi=0 \text { in } \Omega(t) .
$$

Using the free space Green's function, $G\left(\boldsymbol{x}, \boldsymbol{x}_{l}\right)=-(1 / 2 \pi) \log \left|\boldsymbol{x}-\boldsymbol{x}_{l}\right|$, and Green's second identity, equation (17) transforms into the Boundary Integral Equation (BIE),

$$
\alpha\left(\boldsymbol{x}_{l}\right) \phi\left(\boldsymbol{x}_{l}\right)=\int_{\Gamma(\boldsymbol{x})}\left[\frac{\partial \phi}{\partial n}(\boldsymbol{x}) G\left(\boldsymbol{x}, \boldsymbol{x}_{l}\right)-\phi(\boldsymbol{x}) \frac{\partial G\left(\boldsymbol{x}, \boldsymbol{x}_{l}\right)}{\partial n}\right] \mathrm{d} \Gamma(\boldsymbol{x}),
$$

in which $\boldsymbol{x}=(x, z)$ and $\boldsymbol{x}_{l}=\left(x_{l}, z_{l}\right)$ are position vectors for points on the boundary, $\boldsymbol{n}$ is the unit outward normal vector, and $\alpha\left(\boldsymbol{x}_{l}\right)$ is a geometric coefficient function of the exterior angle of the boundary at $\boldsymbol{x}_{l}$.

On the free surface $\Gamma_{f}(t), \phi$ satisfies the kinematic and dynamic boundary conditions,

$$
\begin{array}{ll}
\frac{D \boldsymbol{r}}{D t}=\left(\frac{\partial}{\partial t}+\boldsymbol{u} \cdot \boldsymbol{\nabla}\right) \boldsymbol{r}=\boldsymbol{u}=\boldsymbol{\nabla} \phi & \text { on } \Gamma_{f}(t), \\
\frac{D \phi}{D t}=-g z+\frac{1}{2} \boldsymbol{\nabla} \phi \cdot \nabla \phi-\frac{p_{a}}{\rho} & \text { on } \Gamma_{f}(t),
\end{array}
$$

respectively, with $\boldsymbol{r}$, the position vector on the free surface, $g$ the gravitational acceleration, $z$ the vertical coordinate, $p_{a}$ the pressure at the free surface, and $\rho$ the fluid density. Along the stationary bottom $\Gamma_{b}$, the no-flow condition is prescribed as

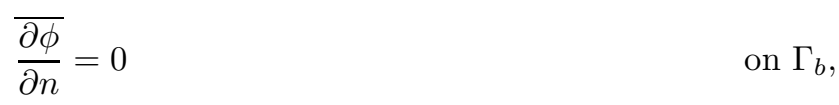

where the overline denotes specified values.

In the present applications, initial solitary waves are directly specified at time $t=0$ on the model free surface $\Gamma_{f}$, using the geometry, potential, and normal gradient of the potential, calculated with Tanaka's method [30], for fully nonlinear solitary waves.

\subsection{Numerical methods}

The BIE (18) is solved by a BEM using $N$ discretization nodes on the boundary and $M$ higher-order elements to interpolate in between discretization nodes. In the present applications, quadratic isoparametric elements are used on lateral and bottom boundaries, and cubic elements ensuring continuity of the boundary slope are used on the free surface. In these elements, referred to as Mixed Cubic Interpolation (MCI) elements, geometry is 
modeled by cubic splines and field variables are interpolated between each pair of nodes, using the mid-section of a four-node "sliding" isoparametric element. Expressions of BEM integrals (regular, singular, quasi-singular) are given in Grilli et al. $[10,14]$ and Grilli and Subramanya [9], for isoparametric and MCI elements.

Free surface boundary conditions (19) and (20) are time integrated in a mixed Eulerian-Lagrangian formulation (MEL) based on two second-order Taylor series expansions expressed in terms of a time step $\Delta t$ and of the Lagrangian time derivative, $D / D t$, for $\phi$ and $\boldsymbol{r}$. First-order coefficients in the series correspond to free surface conditions (19) and (20), in which $\phi$ and $\partial \phi / \partial n$ are obtained from the solution of the BIE for $(\phi, \partial \phi / \partial n)$ at time $t$. Second-order coefficients are expressed as $D / D t$ of equations (19) and (20), and are calculated using the solution of a second BIE for $\left(\partial \phi / \partial t, \partial^{2} \phi / \partial t \partial n\right)$, for which boundary conditions are obtained from the solution of the first problem. Detailed expressions for the Taylor series are given in Grilli et al. [14]. The time step is adaptively calculated at each time step to ensure a mesh Courant number of $C_{o}=0.45$ for the minimum spacing between nodes on the free surface [9], i.e., $\Delta t^{\prime}=C_{o} \Delta x^{\prime}$ (where dashes indicate nondimensional variables, with length divided by $h_{1}$ and time by $\sqrt{h_{1} / g}$ ).

In the MEL formulation, free surface discretization nodes represent fluid particles and, hence, drift in the direction of the mean mass flux of the flow, thereby affecting resolution of the discretization. To either add and redistribute nodes in regions of poor resolution of the free surface or to remove and redistribute nodes in regions of node concentration, a regridding technique was implemented in the model in combination with the MCI interpolation method. This technique redistributes nodes within a specified boundary section, based on a constant arc length interval, or adaptively regrids nodes two by two when they move too close from each other, causing quasi-singularities in the BEM integrals (e.g., in the plunging jet of a breaking wave). Field variables are then re-interpolated using BEM elements, at the new locations of nodes in the regridded section.

The BEM solution for the flow kinematics and pressure is thus computed as a function of time at boundary nodes. Surface piercing numerical wave gages can be specified, at which free surface elevation is calculated as a function of time. Similarly, the model can provide the BEM solution at a specified distribution of (internal) points within the domain (e.g., $[9,18,22])$. These points can be defined either on a fixed grid or on vertical lines, for a number of variable intervals between the free surface and the bottom boundaries. Velocity and pressure computed at such internal points are used to initialize the VOF models, as detailed in [18,22].

\subsection{Results for solitary waves over a step}

Grilli et al. in [12] already compared predictions of a 2D-FNPF BEM model to experiments, for the propagation of solitary waves over a step. They found a very good agreement between these, up to the location of the step. Over the step, however, the FNPF model was found to slightly overpredict wave heights, likely due to energy dissipation resulting from flow separation over the step, not modeled in 2D-FNPFs.

The present computations were run for the case reported in the paper by Yasuda et al. [39], for which these authors both performed laboratory experiments and computations using a potential flow model similar to Dold and Peregrine's (1986). In our computations, for the domain shown in Figure 1, we used 195 MCI elements on the free surface, with spacing $\Delta x^{\prime}=0.1$ between nodes. We used 63 quadratic isoparametric elements on the rest of the boundary, for a total of 326 nodes on the boundary. The initial time step was $\Delta t^{\prime}=0.045$, which gradually reduced as nodes converged in the breaking jet. To mitigate this effect, we used adaptive node regridding on the free surface, with relative distance threshold of 0.5 and 2 . We thus computed 582 varying time steps to reach the last computed surface profile, at $t=1.35 \mathrm{~s}$, just before impact of the plunging jet on the free surface (see Fig. 9). At this time, numerical errors on wave volume and energy are still less than $0.1 \%$, as compared to initial values given by Tanaka's model, for $H_{o}^{\prime}=0.424$. As in our earlier computations, we find very good agreement for wave elevation at the gauge located just at the step (P2; Fig. 5). Beyond the step, however, expectedly, wave elevation is slightly overpredicted (P3; Fig. 6). No adjustments of propagation times were made from the incident wave location at P1 to P3, which, hence, indicates that wave celerity and kinematics predicted in the model are also in agreement with experiments during these stages of propagation. 


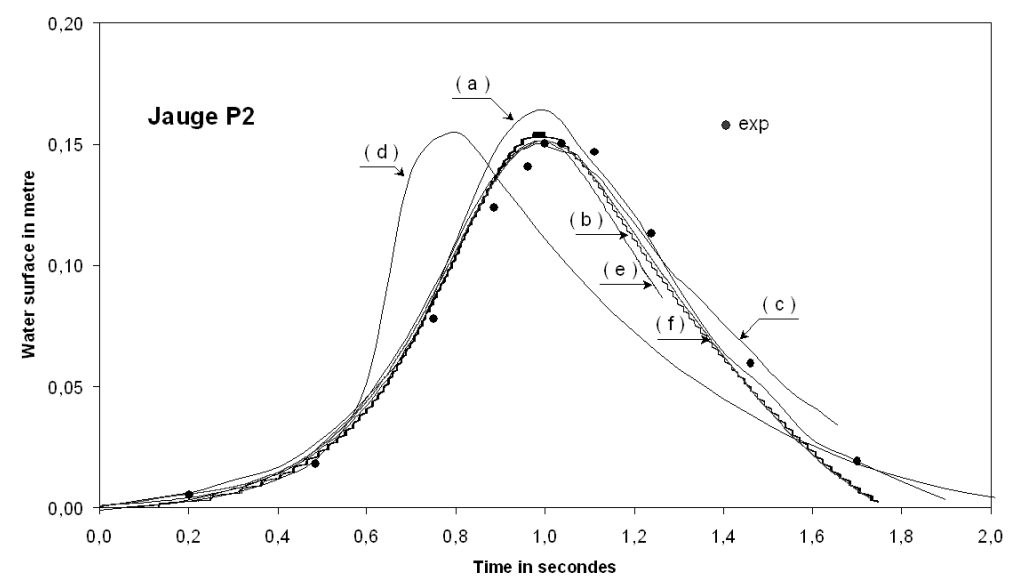

Figure 5. Time evolution of the water surface: gauge P2.

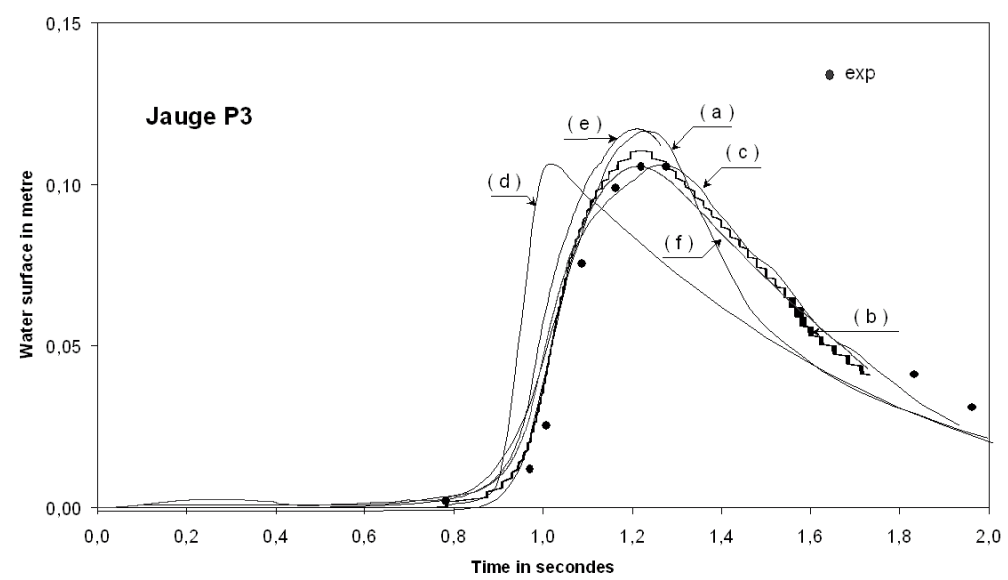

Figure 6. Time evolution of the water surface: gauge P3.

\section{Level-Set method (Lesage, Dervieux, Allain)}

\subsection{Mathematical model}

The computation is performed with a simplified model, i.e. an inviscid incompressible flow with two densities, $\rho_{g}$ for the gas, $\rho_{l}$ for the liquid, and no surface tension. We present this model in combination with a level set formulation:

$$
\left\{\begin{aligned}
\rho(\phi) \frac{D \mathbf{u}}{D t}+\nabla p & =\rho(\phi) \mathbf{g}, \\
\nabla \cdot \mathbf{u} & =0 \\
\frac{\delta \phi}{\delta t}+\mathbf{u} \cdot \nabla \phi & =0
\end{aligned}\right.
$$

where $\rho(\phi)=\rho_{g}+\left(\rho_{l}-\rho_{g}\right) H(\phi), H(x)$ being equal to unity when $x$ is positive, zero else. Symbol u holds for the flow velocity and symbol $\mathbf{g}$ holds for gravity. Typically $\phi$ at time $t=0$ is a signed distance to initial interface. The level-set formulation tends to replace the interface discontinuity by a smooth function easier to advect. 
High-order accuracy for the characteristic function of phases cannot be reached if the level set function involves the too small or too large gradients that can appear after integrating a few time steps. This event is independent of both time and space discretization. We apply the strategy proposed in [6] which consists in replacing the level set after a certain fraction $\frac{T}{n}$ of the total time interval. The new value of level set is a signed distance to interface computed with a geometrical algorithm.

Although not necessary with any Navier-Stokes solver, a further smoothing of the coefficients is generally useful. We give a small thickness to the interface by replacing $H(\phi)$ by:

$$
H^{\epsilon}(\phi)=\frac{1}{2}\left[1+\frac{\phi}{\epsilon}+\frac{1}{\Pi} \sin \left(\frac{\Pi \phi}{\epsilon}\right)\right] \text { if }|\phi| \leq \epsilon, \quad H(\phi) \text { else. }
$$

\subsection{Numerical method}

For the velocity and pressure, this is performed with the classical projection method:

$$
\left\{\begin{aligned}
\nabla \cdot \frac{1}{\rho\left(\phi^{n}\right)} \nabla p^{n+1} & =\nabla\left(\mathbf{u}^{n}-\Delta t h\left(\mathbf{u}^{n}\right)\right), \\
\frac{\mathbf{u}^{n+1}-\mathbf{u}^{n}}{\delta t} & =-h\left(\mathbf{u}^{n}\right)-\frac{\nabla p^{n+1}}{\rho\left(\phi^{n}\right)},
\end{aligned}\right.
$$

with $h\left(\mathbf{u}^{n}\right)=\nabla \cdot\left(\mathbf{u}^{n} \times \mathbf{u}^{n}\right)-\mathbf{g}$.

For the time integration of the level set function equation, we use a third order accurate three-stage RungeKutta time advancing.

For the space integration, we use a mixed element-volume method. A continuous piecewise-linear finite element Galerkin approximation is applied to all terms of the model except the convective velocity terms and the advection of the level set function. For those convective and advective terms, we apply an upwind finitevolume method based on cells built from element medians. It is upwind-biased and third-order accurate for linear advection. We use an uniform 600000 cells 3D mesh for $t \in[0,1 \mathrm{~s}]$. For $t \in[1 \mathrm{~s}, 1.8 \mathrm{~s}]$, we make a mesh adaptation thanks to an interpolation of the numerical result at $t=1 \mathrm{~s}$ on a non-uniform 600000 cells $3 \mathrm{D}$ mesh refined in the breaking area.

\section{Comparison of numerical Results}

In this section, we compare the results obtained with the six different methods. The methods will be shortly referred as follows:

- method (a): the compressible solver (Helluy, Golay);

- method (b): the incompressible solver (Caltagirone, Lubin, Vincent);

- method (c): the VOF solver (Drevard, Marcer, Fraunié);

- method (d): the shallow-water solver (Séguin);

- method (e): the boundary integral solver (Grilli);

- method (f): the level-set solver (Lesage, Dervieux, Allain).

\subsection{Computation data}

The Table 1 give the characteristics of the different computations.

\subsection{Comparison with experiments}

In order to assess our numerical results, we compared them with the experimental measurements of Yasuda, Mutsuda and Mizutani in [39]. The first measurements deal with the evolution of the water surface during time at the gauges P2, P3 and P4 (see Fig. 1). The experimental and numerical results are compared in Figures 5, 6 and 7 . 
TABLE 1. Computation costs.

\begin{tabular}{|c|c|c|c|c|}
\hline Case & cells in $x$ & cells in $y$ & time & CPU type \\
\hline (a) & 1500 & 200 & $48 \mathrm{~h}$ & Alpha 666 Mhz 64 bits \\
\hline (b) & 1200 & 200 & $76 \mathrm{~h}$ & Itanium 1.4 Ghz 64 bits \\
\hline (c) & 1000 & 200 & $29 \mathrm{~h}$ & Alpha 666 Mhz 64 bits \\
\hline (d) & 500 & none & $1 \mathrm{~min}$ & Pentium 1 Ghz 32 bits \\
\hline (e) & 326 & none & $8 \mathrm{~min}$ & PowerPC G4 1.2 Ghz 32 bits \\
\hline (f) 3D & 1500 & 200 & $72 \mathrm{~h}$ & Pentium 4 $3 \mathrm{Ghz} 32 \mathrm{bits}$ \\
\hline
\end{tabular}

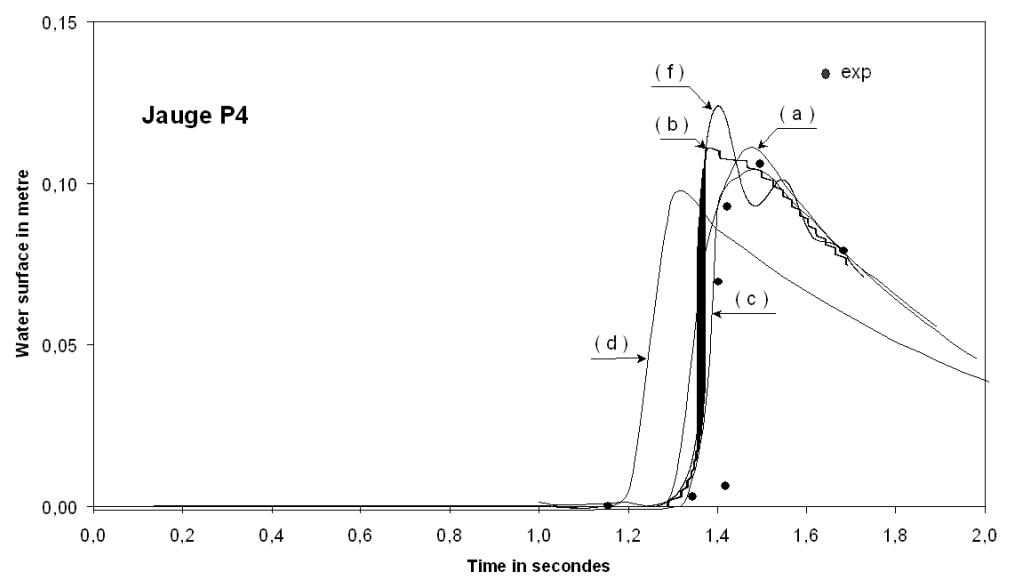

FIGURE 7. Time evolution of the water surface: gauge P4.

We can make the following comments:

- The shallow water model (d) clearly gives the worst results in all the cases: the waves are shifted in time and the amplitudes are lower than the experimental ones. Let us recall however that the computational cost is very low. The results could be improved by adding dispersive third order terms in the model.

- At the gauge P2 (Fig. 5), maybe due to an artificial compressibility effect, the compressible model (a) slightly overpredicts the wave height. Let us recall that the preconditioning of this solver is very rough. Maybe that a more sophisticated preconditioning would have improved the precision. The other methods (b, c, e, f) give more or less the same results and are very close to the experiments.

- At the gauge P3 (Fig. 6), the wave height is overpredicted at the highest point by the compressible model (a), then it is underpredicted. We observe also an overprediction by the boundary integral model (e). It is likely due to energy dissipation resulting from flow separation over the step, not modeled in a potential flow. The other methods (b, c, f) give more or less the same results and are very close to the experiments.

- At the gauge P4 (Fig. 7) we observe time shifts in the incompressible and level-set models (b, f). We observe slight oscillations of the water elevation in the level-set model (f). It is now the compressible model (a) and the VOF model (c) that are closer to experiments. 


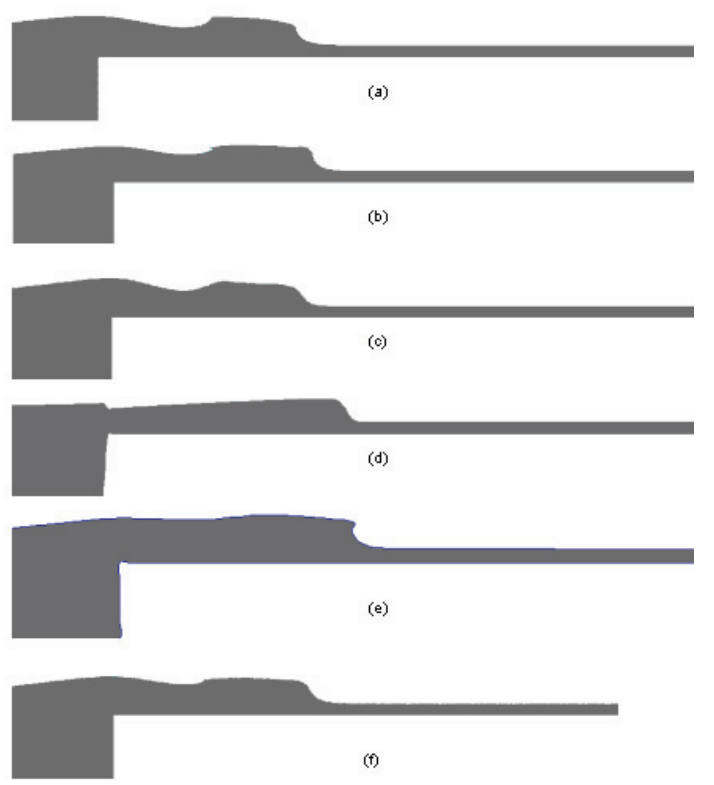

Figure 8. Free surface profiles at $t=1.2 \mathrm{~s}$.

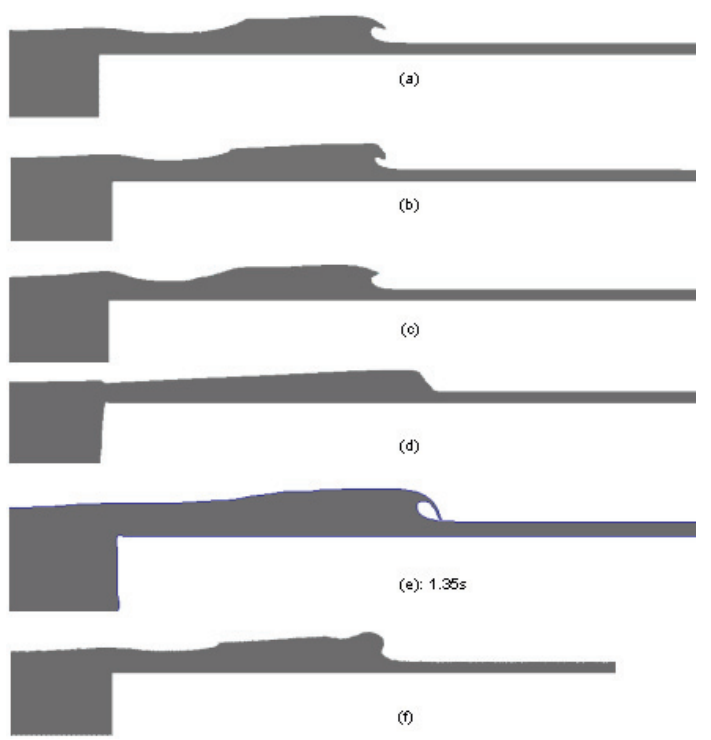

Figure 9. Free surface profiles at $t=1$.4s. Warning: the profile (e) is plotted at time $t=1.35 \mathrm{~s}$.

\subsection{Wave profiles}

Then we compare the numerical profiles obtained by the previous methods at time $t=1.2 \mathrm{~s}, t=1.4 \mathrm{~s}$, $t=1.6 \mathrm{~s}$ and $t=1.8 \mathrm{~s}$. The numerical profiles for the 6 methods are compared on Figures 8-11. Here, no experimental results were available and we observe some differences between the 6 methods, indicating that a further study would be interesting. The following remarks can be stated: 


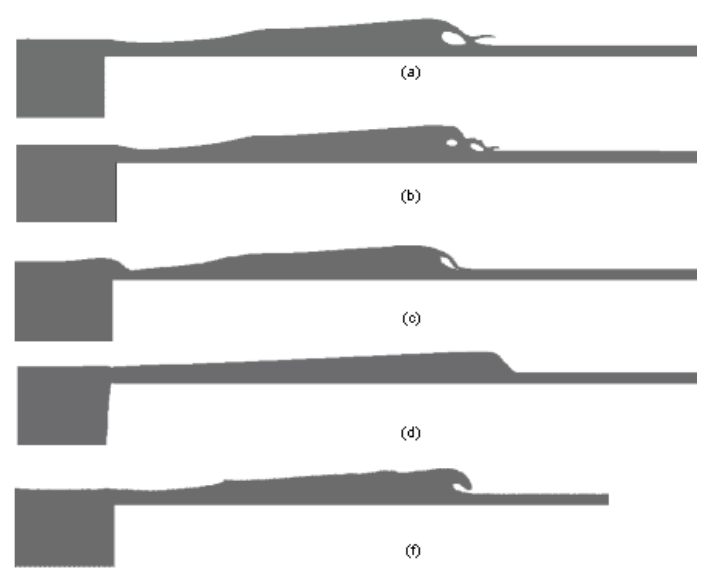

Figure 10. Free surface profiles at $t=1.6 \mathrm{~s}$.

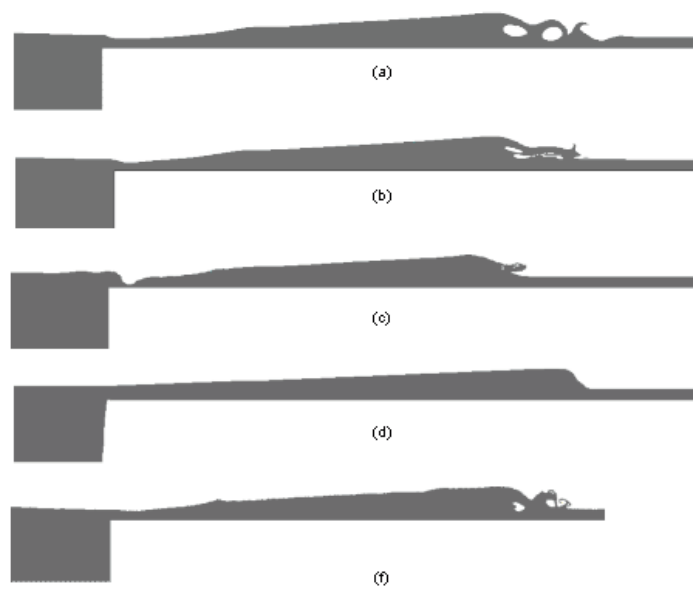

Figure 11. Free surface profiles at $t=1.8 \mathrm{~s}$.

- The boundary integral method (e) is not well adapted to this case because of the non-smooth geometry of the reef. The overprediction of the wave heights implies an exaggeration of the wave speed and the breaking occurs too early. The results would have been much more precise for a smooth reef. With the boundary integral method (e), let us recall that the computation cannot be continued after the reconnection.

- The compressible, incompressible, VOF and level-set solvers (a, b, c, f) give more or less the same wave profiles at time $t=1.2 \mathrm{~s}$ (Fig. 8), with slight differences.

- At times $t=1.4 \mathrm{~s}$, the wave profiles start to be very different. The profile given by the level-set method (f) is not realistic. Maybe the (non-uniform) mesh should have been refined.

- At times $t=1.6 \mathrm{~s}$, the wave profiles are quantitatively very different between all the methods.

- At times $t=1.8 \mathrm{~s}$, the incompressible, VOF and level-set solver (b, c, f) show a turbulent-like behavior. Except for the compressible solver (a), we observe an excessive damping of the wave. 


\section{CONCLusion}

We have compared in this paper several numerical methods to compute the wave breaking of a solitary wave over a reef.

Before the reconnection of the jet, the several methods give more or less the same results. In particular, the agreement with the experimental gauges measurements is satisfactory. This indicates that the incompressible mathematical model is justified in this case. This also justify the several numerical methods. The preconditioned compressible solver is interesting because the method is very simple to implement (no implicit scheme, no particular treatment of the interface) and the cost is comparable, for a given precision, to the incompressible solvers. The precision could be improved by a more sophisticated preconditioner in order to compete with the best incompressible solvers.

Approaching the reconnection, several quantitative differences start to appear. It would be interesting to perform now larger computations, with finer meshes in order to verify that the different methods "converge" towards the same solution. It would have been also interesting to compare the velocity fields between the methods. All the numerical results are collected on the web site of the workshop: http://helluy . univ-tln.fr/soliton.htm.

After the reconnection, different behaviors are observed between the different methods. Here also, a further study is necessary to explain the differences. What are the influences of surface tension, viscosity (real or numerical), turbulence, compressibility, precision etc.? what happens with VOF methods or multiphase models when the topology of the interface becomes more complex?

It also appears, but it is not really a surprise, that the most realistic models are also the most expensive. It is possible that the $3 \mathrm{D}$ effects become more and more important after the reconnection. The current power of personal computers is not sufficient to envisage precise 3D computations (for a recent attempt, see [2,3,24]). Recall that these numerical simulations can be interesting for the calibrating of empirical models in coastal engineering.

\section{REFERENCES}

[1] T. Barberon, P. Helluy and S. Rouy, Practical computation of axisymmetrical multifluid flows. Internat. J. Finite Volumes 1 (2003) 1-34.

[2] B. Biausser, S.T. Grilli and P. Fraunié, Numerical Simulations of Three-dimensional Wave Breaking by Coupling of a VOF Method and A Boundary Element Method, in Proc. 13th Offshore and Polar Engrg. Conf., ISOPE03, Honolulu, USA (May 2003) 333-339.

[3] B. Biausser, S. Guignard, R. Marcer and P. Fraunié, 3D two phase flows numerical simulations by SL-VOF method. Internat. J. Numer. Methods Fluids 45 (2004) 581-604.

[4] J.U. Brackbill, B.D. Kothe and C. Zemach, A continuum method for modeling surface tension. J. Comput. Phys. 100 (1992) $335-354$.

[5] C. De Jouëtte, H. Viviand, S. Wormon and J.M. Le Gouez, Pseudo compressibility method for incompressible flow calculation, in 4 th Int. Symposium on computational Fluid Dynamics, Davis, California, 9-12 September (1991).

[6] A. Dervieux, Résolution de problèmes à frontière libre. Thesis, Paris VI (1981).

[7] T. Gallouët, J.-M. Hérard and N. Seguin, Some approximate godunov schemes to compute shallow-water equations with topography. Comput. Fluids 32 (2003) 479-513.

[8] S.T. Grilli, Fully Nonlinear Potential Flow Models used for Long Wave Runup Prediction, in Long-Wave Runup Models, H. Yeh, P. Liu and C. Synolakis Eds., World Scientific Pub (1997) 116-180.

[9] S.T. Grilli and R. Subramanya, Numerical modeling of wave breaking induced by fixed or moving boundaries. Comput. Mech. 17 (1996) 374-391.

[10] S.T. Grilli and I.A. Svendsen, Corner problems and global accuracy in the boundary element solution of nonlinear wave flows. Engrg. Analysis Boundary Elements 7 (1990) 178-195.

[11] S.T. Grilli, P. Guyenne and F. Dias, A fully nonlinear model for three-dimensional overturning waves over arbitrary bottom, Internat. J. Numer. Methods Fluids 35 (2001) 829-867.

[12] S.T. Grilli, M.A. Losada and F. Martin, The Breaking of a Solitary Wave over a Step: Modeling and Experiments, in Proc. 4 th Intl. Conf. on Hydraulic Engineering Software (HYDROSOFT92, Valencia, Spain, July 92), W.R. Blain and E. Cabrera Eds., Elsevier, Applied Science, Fluid Flow Modelling, Computational Mechanics Publications 1992575-586 (1992).

[13] S.T. Grilli, M.A. Losada and F. Martin, Characteristics of solitary wave breaking induced by breakwaters, J. Waterway Port Coastal Ocean Engrg. 120 (1994) 74-92. 
[14] S.T. Grilli, J. Skourup and I.A. Svendsen, An Efficient Boundary Element Method for Nonlinear Water Waves. Engrg. Analysis Boundary Elements 6 (1989) 97-107.

[15] S.T. Grilli, I.A. Svendsen and R. Subramanya, Breaking criterion and characteristics for solitary waves on slopes. J. Waterway Port Coastal Ocean Engrg. 123 (1997) 102-112.

[16] S.T. Grilli, I.A. Svendsen and R. Subramanya, Closure of: Breaking criterion and characteristics for solitary waves on slopes. J. Waterway Port Coastal Ocean Engrg. 124 (1997) 333-335.

[17] S.T. Grilli, R. Subramanya, I.A. Svendsen and J. Veeramony, Shoaling of solitary waves on plane beaches. J. Waterway Port Coastal Ocean Engrg. 120 (1994) 609-628.

[18] S. Guignard, S.T. Grilli, R. Marcer and V. Rey, Computation of Shoaling and Breaking Waves in Nearshore Areas by the Coupling of BEM and VOF Methods, in Proc. 9th Offshore and Polar Engng. Conf., ISOPE99, Brest, France 3 (May 1999) 304-309.

[19] S. Guignard, R. Marcer, V. Rey, C. Kharif and P. Fraunié, Solitary wave breaking on sloping beaches: 2D two phase flow numerical simulation by SL-VOF method. Eur. J. Mech. B Fluids 20 (2001) 57-74.

[20] H. Guillard and C. Viozat, On the behavior of upwind schemes in the low Mach number limit. Comput. Fluids 28 (1999) 63-86.

[21] C.W. Hirt and B.D. Nichols, Volume of fluid method for the dynamics of free boundaries. J. Comput. Phys. 39 (1981) $323-345$.

[22] C. Lachaume, B. Biausser, S.T. Grilli, P. Fraunié and S. Guignard, Modeling of Breaking and Post-breaking Waves on Slopes by Coupling of BEM and VOF methods, in Proc. 13th Offshore and Polar Engng. Conf., ISOPE03, Honolulu, USA (May 2003) 353-359.

[23] J. Li, Piecewise linear interface calculation. Technical report, Fascicule B-Mecanique, C. R. Acad. Sci. Paris Ser. II. (1995).

[24] P. Lubin, S. Vincent, J. Caltagirone and S. Abadie, Fully three-dimensional numerical simulation of a plunging breaker. C. R. Mécanique 331 (2003) 495-501.

[25] P. Lubin, S. Vincent, J. Caltagirone and S. Abadie, Large eddy simulation of vortices induced by plunging breaking waves, in Proc. ISOPE 2004, 14th Intl. Offshore and Polar Enginering Conference and Exhibition 3 (2004) 306-312.

[26] S. Osher and R. Fedkiw, Level Set Methods and Dynamic Implicit Surfaces. Springer-Verlag, New York (2002).

[27] P. Sagaut, Large eddy simulation for incompressible flows. Springer-Verlag, New York (1998).

[28] R. Saurel and R. Abgrall, A simple method for compressible multifluid flows. SIAM J. Sci. Comput. 21 (1999) 1115-1145.

[29] J.A. Sethian, Level Set Methods: Evolving Interfaces in Geometry, Fluid Mechanics, Computer Vision and Materials Sciences. Cambridge University Press (1996).

[30] M. Tanaka, The stability of solitary waves. Phys. Fluids 29 (1986) 650-655.

[31] E. Turkel, Preconditioned methods for solving the incompressible and low speed compressible equations. J. Comput. Phys. 72 (1987) 277-298.

[32] E. Turkel, Review of preconditioning methods for fluid dynamics. Appl. Numer. Math. 12 (1993) 257-284.

[33] S. Vincent, Modélisation d'écoulements incompressibles de fluides non-miscibles. Université Bordeaux I (1999).

[34] S. Vincent and J.P. Caltagirone, Efficient solving method for unsteady incompressible interfacial flow problems. Internat. J. Numer. Methods Fluids 30 (1999) 795-811.

[35] S. Vincent and J.P. Caltagirone, A one cell local multigrid method for solving unsteady incompressible multi-phase flows. J. Comput. Phys. 163 (2000) 172-215.

[36] S. Vincent, J.P. Caltagirone, P. Lubin and T.N. Randrianarivelo, an adaptative augmented Lagrangian method for threedimensional multi-material flows. Comput. Fluids (2004), under press.

[37] H. Viviand, Pseudo-unsteady methods for transonic flow computations, in 19th Int. Conf. on Numerical Methods in Fluid Dynamics, Stanford, Springer-Verlag, New-York 141 (1980).

[38] H. Viviand, Analysis of pseudo-compressibility systems for compressible and incompressible flows. Technical report, Comput. Fluids Dynamics Rev. (1995).

[39] T. Yasuda, H. Mutsuda and N. Mizutani, Kinematic of overtuning solitary waves and their relations to breaker types. Coastal Engrg. 29 (1997) 317-346. 